\title{
The long-time dynamics of 3D non-autonomous Navier-Stokes equations with variable viscosity
}

\author{
Yongqiang Chen, Xin-guang Yang*, Mengmeng Si \\ Department of Mathematics and Information Science, Henan Normal University, Xinxiang, 453007, China
}

*Corresponding author, e-mail: yangxinguang@hotmail.com

Received 11 Jun 2017

Accepted 15 Oct 2017

\begin{abstract}
This paper is concerned with the long-time dynamics of some 3D generalized non-autonomous NavierStokes equations in a bounded domain governing the motion of fluid flow which comes from a monograph by Ladyzhenskaya. Under some assumptions on the external force and initial data, we prove the existence and structure of uniform attractors for the continuous process.
\end{abstract}

KEYWORDS: translation bounded functions, uniform attractors

MSC2010: 35Q30 35B40 35B41 76D03 76D05

\section{INTRODUCTION}

The objective of this paper is to study the uniform attractor of the 3D incompressible modified NavierStokes equations,

$$
\left.\begin{array}{rl}
u_{t}-\left(v_{0}+v_{1}\|u\|^{2}\right) \Delta u+(u \cdot \nabla) u+\nabla p & =\sigma(t, x), \\
\nabla \cdot u=0, & \\
\left.u\right|_{\partial \Omega} & =0, \\
u(x, \tau) & =u_{0}(x),
\end{array}\right\}
$$

defined in a bounded domain $\Omega \subset \mathbb{R}^{3}$, with the smooth boundary $\partial \Omega$, where $u$ is the velocity field with components $u^{i}=u^{i}(t, x), i=1,2,3$, and $p=$ $p(t, x)$ is the pressure. The assumption of nonlinear viscosity $v=v_{0}+v_{1}\|u\|^{2}$, where $v_{0}, v_{1}>0$ and was proposed in Ref. 1 . If the viscosity $v(t)>0$ becomes a constant, (1) reduces to the well-known classical 3D Navier-Stokes equations.

The classical Navier-Stokes equations ( $v$ is a constant) are an important part of the theory for fluid mechanics ${ }^{1-5}$. A comprehensive description of the theory of the Navier-Stokes equations can be found in Refs. 6-8.

For the variable viscosity problem (1), the background of this model and some existence results can be founded in Ref. 1. The uniqueness of this problem was investigated by Ref. 9 with $N<3$ spatial dimensions. This equation was also considered in Ref. 10, where the existence of weak solutions was obtained for $N \leqslant 4$ and uniqueness for $N \leqslant 3$, and the well-posedness of (1) in a non-cylindrical domain ${ }^{11}$. For the pullback dynamics of system (1) and its modified form, see Refs. 12,13. However, as far as we know, there is no result about the forward dynamics of this model with some non-autonomous external forces. We shall use the energy equation method to deal with the uniformly asymptotic compactness of continuous processes with respect to the special external force (which is called a symbol) for achieving the existence of uniform attractors.

After introducing the notation and some preliminaries, the well-posedness of problem (1) will be shown. We then gives the theory of uniform attractors and the main results.

\section{NOTATION AND PRELIMINARIES}

Throughout this paper, the Hausdorff distance in $X$ from one set $B_{1}$ to another $B_{2}$ is defined as

$$
\operatorname{dist}_{X}\left(B_{1}, B_{2}\right)=\sup _{b_{1} \in B_{1}} \inf _{b_{2} \in B_{2}}\left\|b_{1}-b_{2}\right\|_{X} .
$$

Setting $E:=\left\{u \mid u \in\left(C_{0}^{\infty}(\Omega)\right)^{3}\right.$, divu $\left.=0\right\}, H$ is the closure of $E$ with $\left(L^{2}(\Omega)\right)^{3}$ topology, and $V$ is the closure of $E$ in $\left(H^{1}(\Omega)\right)^{3}$ topology. $|\cdot|$ and $(\cdot, \cdot)$ denote the norm and inner product of $H$. Here,

$$
(u, v)=\sum_{j=1}^{3} \int_{\Omega} u_{j}(x) v_{j}(x) \mathrm{d} x, \quad \forall u, v \in H .
$$

$\|\cdot\|$ and $((\cdot, \cdot))$ denote the norm and inner product in $V$. Here,

$$
((u, v))=\sum_{i, j=1}^{3} \int_{\Omega} \frac{\partial u_{j}}{\partial x_{i}} \frac{\partial v_{j}}{\partial x_{i}} \mathrm{~d} x, \quad \forall u, v \in V .
$$


Clearly, $V \hookrightarrow H \equiv H^{\prime} \hookrightarrow V^{\prime}, H^{\prime}$ and $V^{\prime}$ are the dual spaces of $H$ and $V$, respectively, where the injection is dense and continuous. $\|\cdot\|_{*}$ denotes the norm of $V^{\prime}$, and $\langle\cdot\rangle$ is the dual product between $V$ and $V^{\prime}$.

Let $P$ be the Helmholtz-Leray orthogonal projection operator from $\left(L^{2}(\Omega)\right)^{3}$ onto $H$. We define $A:=$ $-P \Delta$ as the Stokes operator with domain $D(A)=$ $\left(H^{2}(\Omega)\right)^{3} \cap\left(H_{0}^{1}(\Omega)\right)^{3}$ and $\lambda$ is the first eigenvalue of $A$, then the operator $A: V \rightarrow V^{\prime}$ has the property $\langle A u, v\rangle=((u, v))$ for all $u, v \in V$ which is an isomorphism from $V$ into $V^{\prime}$, and $\left\{\lambda_{j}\right\}_{j=1}^{\infty}$ for $0<\lambda_{1} \leqslant$ $\lambda_{2} \leqslant \cdots$ are eigenvalues of $A$ corresponding to the eigenfunctions $\left\{\omega_{j}\right\}_{j=1}^{\infty}$, i.e., $A \omega_{j}=\lambda_{j} \omega_{j}$.

We define the bilinear operator and trilinear operator as ${ }^{6,14}$

$$
\begin{gathered}
B(u, v):=P((u \cdot \nabla) v), \quad \forall u, v \in E \\
b(u, v, w)=(B(u, v), w)=\sum_{i, j=1}^{3} \int_{\Omega} u_{i} \frac{\partial v_{j}}{\partial x_{i}} w_{j} \mathrm{~d} x
\end{gathered}
$$

which satisfy

$$
\begin{gathered}
b(u, v, v)=0, b(u, v, w)=-b(u, w, v), \\
\forall u, v, w \in V, \\
|b(u, v, w)| \leqslant C|u|^{1 / 2}\|u\|_{V}^{1 / 2}\|v\|_{V}|w|^{1 / 2}\|w\|_{V}^{1 / 2}, \\
\forall u, v, w \in V .
\end{gathered}
$$

\section{THE GLOBAL EXISTENCE OF SOLUTIONS}

The operator $\mathbb{A}: V \rightarrow V^{\prime}$ is defined by $\mathbb{A} u=$ $-v_{1}\|u\|^{2} \Delta u$ and

$$
\begin{aligned}
\langle\mathbb{A} u, v\rangle & =v_{1}\|u\|^{2}\langle-\Delta u, v\rangle \\
& =v_{1}\|u\|^{2}((u, v)), \quad \forall u, v \in V .
\end{aligned}
$$

Noting that $\mathbb{A}$ maps the bounded sets in $V$ to bounded sets in $V^{\prime}$, we have

$$
\begin{aligned}
\|\mathbb{A}\|_{V^{\prime}} & =\sup _{\|v\|=1, v \in V}|\langle\mathbb{A} u, v\rangle| \\
& =\sup _{\|v\|=1, v \in V} v_{1}\|u\|^{2}|a(u, v)| \leqslant v_{1}\|u\|^{3} .
\end{aligned}
$$

We call the function $u(t, x) \in L^{\infty}(\tau, T ; H) \cap$ $L^{4}(\tau, T ; V)$ a weak solution of (1) if

$$
\begin{aligned}
& \frac{\mathrm{d}}{\mathrm{d} t}(u(t), v)+\left(v_{0}+v_{1}\|u(t)\|^{2}\right)((u(t), v)) \\
& \quad+b(u, u, v)=\langle g(t, x), v\rangle, \quad u(\tau)=u_{0}, \tau \in \mathbb{R},
\end{aligned}
$$

for $v \in V$ in distributed sense.

Based on the above definition and notation, (1) can be rewritten as an equivalent abstract functional equation:

$$
u_{t}+v_{0} A u+\mathbb{A} u+B(u, u)=f(t, x), \quad \nabla \cdot u=0 .
$$

The existence, uniqueness and regularity of a global solution for (5) can be derived by the Galerkin approximation method and some energy estimates. Here we omit the details; the technique can be found in Refs. 9, 10.

Theorem 1 (a) Assume the external force $f(t, x) \in$ $L_{\text {loc }}^{4 / 3}\left(\mathbb{R}, V^{\prime}\right)$ and initial data $u_{0} \in H$. Then the system (5) has a unique weak solution

$$
u(x, t) \in L^{\infty}(\tau, T ; H) \cap L^{4}(\tau, T ; V)
$$

in dimension $N \leqslant 3$. (b) Furthermore, since the solution has continuous dependence on the initial data and $(\mathrm{d} u / \mathrm{d} t) \in L^{2}\left(\tau, T ; V^{\prime}\right)$, from the Aubin-Lions Lemma, we derive that $u(t, x) \in C(\tau, T ; H)$ which generates a continuous process $U(t, \tau): H \rightarrow H$.

\section{THE THEORY OF UNIFORM ATTRACTORS}

We firstly recall some theory of uniform attractors ${ }^{15,16}$. Let $X$ be a Banach space with a spatial variable. The space $L_{\text {loc }}^{p}(\mathbb{R} ; X)$ denotes all functions with spatial values in a Banach space $X$ and time variable locally $p$-power integrable in the Bochner sense with the norm $\int_{t_{1}}^{t_{2}}\|\cdot\|_{X}^{p} \mathrm{~d} s<\infty$ for any time interval $\left[t_{1}, t_{2}\right] \subseteq \mathbb{R}$. We choose $\Sigma=\mathscr{H}\left(\sigma_{0}\right)$ as a symbol space of the system,

$$
\mathscr{H}\left(\sigma_{0}\right)={\overline{\left\{\sigma_{0}(\cdot+h) \mid h \in \mathbb{R}^{+}\right.}}^{\mathscr{E}}
$$

for every fixed $\sigma_{0} \in \mathscr{E}$. Let $\{T(\cdot)\}$ be the translation semigroup (also the shift operator) defined on $\Sigma$ (the symbol space): $T(\cdot) \sigma(s)=\sigma(s+\cdot)$ and the symbol space is invariant in the sense $T(\cdot) \Sigma=\Sigma$. The space $L_{b}^{2}(\mathbb{R} ; X)$ denotes the union of all translation bounded functions satisfying

$$
\|\sigma\|_{L_{b}^{2}(\mathbb{R} ; X)}^{2}=\sup _{t \in \mathbb{R}} \int_{t}^{t+1}\|\sigma(s)\|_{X}^{2} \mathrm{~d} s<\infty,
$$

for $\sigma \in L_{b}^{2}(\mathbb{R} ; X)$. We say $\sigma \in L_{\text {loc }}^{2}(\mathbb{R} ; X)$ is translation compact in $L_{\text {loc }}^{2}(\mathbb{R} ; X)$ if $\mathscr{H}(\sigma)$ is compact in $L_{\text {loc }}^{2}(\mathbb{R} ; X)$ which is denoted by $L_{c}^{2}(\mathbb{R} ; X) . L_{c}^{2, w}(\mathbb{R} ; X)$ is the weak translation compact space. We define the normal functional space $L_{n}^{2}(\mathbb{R} ; X)$ as there exists a $\eta>0$, such that for any $\varepsilon>0$

$L_{n}^{2}(\mathbb{R} ; X)=\left\{\phi \in L_{\text {loc }}^{2}(\mathbb{R} ; X): \sup _{t \in \mathbb{R}} \int_{t}^{t+\eta}\|\phi(s)\|_{X}^{2} \mathrm{~d} s \leqslant \varepsilon\right\}$.

Remark 1 If we choose $\sigma_{0} \in L_{\text {loc }}^{2}(\mathbb{R} ; X)$ and fixed, then for every $\sigma \in \mathscr{H}\left(\sigma_{0}\right)$, we have

$$
\begin{gathered}
\|\sigma\|_{L_{b}^{2}(\mathbb{R} ; X)}^{2} \leqslant C\left\|\sigma_{0}\right\|_{L_{b}^{2}(\mathbb{R} ; X)}^{2}, \\
L_{c}^{2}(\mathbb{R} ; X) \subset L_{n}^{2}(\mathbb{R} ; X) \subset L_{b}^{2}(\mathbb{R} ; X) \subset L_{\mathrm{loc}}^{2}(\mathbb{R} ; X) .
\end{gathered}
$$


Denoting the phase space of global solutions as $E$, we can define $\left\{U_{\sigma}(t, \tau)\right\}: E \rightarrow E$ as evolutionary processes if

$$
\begin{aligned}
U_{\sigma}(t, s) U_{\sigma}(s, \tau) & =U_{\sigma}(t, \tau), \quad \forall t \geqslant s \geqslant \tau, \tau \in \mathbb{R}, \\
U_{\sigma}(\tau, \tau) & =\operatorname{Id} \text { (identity), } \quad \forall \tau \in \mathbb{R}
\end{aligned}
$$

for any function $\sigma \in \Sigma, \Sigma \subset \mathscr{E}$. Furthermore, we assume that the family of processes $\left\{U_{\sigma}(t, \tau)\right\}$ satisfies the translation identity for invariance

$$
\begin{aligned}
& U_{\sigma(t)}(t+h, \tau+h)=U_{T(h) \sigma(t)}(t, \tau), \\
& \forall \sigma \in \Sigma, t \geqslant \tau, \tau, s \in \mathbb{R} .
\end{aligned}
$$

We define the skew product flow as $S(t)=$ $\left(U_{\sigma}(t, \tau), T(t)\right) \in E \times \Sigma$, i.e.,

$$
\begin{aligned}
S(t)(u(s), \sigma(s)) & =\left(U_{\sigma(s)}(t, 0) u(s), T(t) \sigma(s)\right) \\
& =(u(t+s), \sigma(t+s))
\end{aligned}
$$

for every $(u(s) \sigma(s)) \in E \times \Sigma$.

Remark 2 If the family of processes satisfies the translation identity (6) and it is $(E \times \Sigma, \Sigma)$ continuous, then the skew product flow is a semigroup.

Theorem 2 (Ref. 15) Assume that (i) $\Sigma$ is the symbol space and it is invariant under the continuous translation semigroup $\{T(h)\}$ defined above; (ii) the family of processes $\left\{U_{\sigma}(t, \tau)\right\}, \sigma \in \Sigma$ acting on the phase space $E$ which satisfies the translation identity (6) is uniformly asymptotically compact and $(E \times \Sigma, E)$-continuous; (iii) there exists a uniformly bounded absorbing set $B$ with respect to every symbol $\sigma \in \Sigma$

Let $\Pi_{1}$ and $\Pi_{2}$ be two projectors from $E \times \Sigma$ onto $E$ and $\Sigma$, respectively: $\Pi_{1}(u, \sigma)=u$ and $\Pi_{2}(u, \sigma)=$ $\sigma$. Then the skew product flow possesses a global attractor $\mathscr{A}$.

A curve $u(s), s \in \mathbb{R}$ is called the complete trajectory for the process $\{U(t, \tau)\}$ if $U(t, \tau) u(\tau)=u(t)$ for all $t \geqslant \tau, \tau \in \mathbb{R}$. The kernel $\mathscr{K}$ of the process $\{U(t, \tau)\}$ is defined as all bounded complete trajectories: $\mathscr{K}=\{u(\cdot) \mid u(\cdot)$ are complete trajectories and $\left.\|u(\cdot)\|_{E} \leqslant C\right\}$. The kernel section $\mathscr{K}(s)=\{u(s) \mid$ $u(\cdot) \in \mathscr{K}\}$ is the kernel at time $t=s$.

Theorem 3 (Ref. 15) The compact global attractor has the structure $\mathscr{A}=\bigcup_{\sigma \in \Sigma} \mathscr{K}_{\sigma}(\tau) \times\{\sigma\}$ which is (a) strictly invariant $(S(t) \mathscr{A}=\mathscr{A})$ and (b) satisfies $\Pi_{1} \mathscr{A}=\mathscr{A}_{\Sigma}=\bigcup_{\sigma \in \Sigma} \mathscr{K}_{\sigma}(\tau)$ is the uniform attractor for $\left\{U_{\sigma}(t, \tau)\right\}, \Pi_{2} \mathscr{A}=\Sigma$, where $\mathscr{K}_{\sigma}(\tau)$ is the section at $t=\tau$ of the kernel $\mathscr{K}_{\sigma}$ for the process $\left\{U_{\sigma}(t, \tau)\right\}$ with symbol $\sigma \in \Sigma$.

\section{THE UNIFORM ATTRACTOR FOR 3D NAVIER-STOKES EQUATIONS WITH VARIABLE VISCOSITY}

\section{Symbol space}

We denote $E_{1}=L_{\text {loc }}^{2}\left(\mathbb{R} ;\left(L^{2}(\Omega)\right)^{2}\right)$ as the 2-power locally integrable functional space, and define

$$
\begin{gathered}
\hat{E}_{1}=L_{b}^{2}\left(\mathbb{R} ;\left(L^{2}(\Omega)\right)^{2}\right), \quad \bar{E}_{1}=L_{c}^{2}\left(\mathbb{R} ;\left(L^{2}(\Omega)\right)^{2}\right), \\
\tilde{E}_{1}=L_{n}^{2}\left(\mathbb{R} ;\left(L^{2}(\Omega)\right)^{2}\right)
\end{gathered}
$$

as translation bounded, translation compact, and normal functional spaces, respectively.

Choosing an arbitrary function $\sigma_{0} \in \bar{E}_{1}, \hat{E}_{1}$, or $\tilde{E}_{1}$, then we can define the symbol space $\mathscr{H}\left(\sigma_{0}\right)$ which is called the hull of $\sigma_{0}$ by

$$
\bar{\Sigma}=\mathscr{H}\left(\sigma_{0}\right)=\left[\sigma_{0}(t+h)=T(h) \sigma_{0}(t) \mid h \in \mathbb{R}^{+}\right]_{E_{1}},
$$

where $[\cdot]_{E_{1}}$ denotes the closure in the strong topology of $E_{1}$, and $T(\cdot)$ denotes the translation semigroup.

Proposition 1 (Ref. 15) For every fixed function $\sigma_{0}(\cdot)$ and $\sigma(\cdot)$ belonging to its corresponding symbol space, we have $\|\sigma\|_{L_{b}^{2}\left(\mathbb{R} ; L^{2}(\Omega)\right)}^{2} \leqslant C\left\|\sigma_{0}\right\|_{L_{b}^{2}\left(\mathbb{R} ; L^{2}(\Omega)\right)^{2}}^{2}$

\section{The skew product flow and its global attractors}

The problem (1) possesses a unique global weak solution in $H$. Thus for any symbol $\sigma(\cdot) \in \Sigma_{0}$ and $u_{\tau} \in H$, the unique solution generates the process $U_{\sigma(\cdot)}(t, \tau): H \rightarrow H$ as

$$
U_{\sigma(\cdot)}(t, \tau) u_{\tau}=u\left(t, \tau ; u_{\tau}\right) \in H
$$

which satisfies the translation identity.

Choosing the fixed symbol space $\Sigma$ which can be any one of $\bar{E}_{1}, \hat{E}_{1}$ or $\tilde{E}_{1}$, we can derive the skew product flow $S(t)=\left(U_{f(\cdot)}(t, \tau), T(\cdot)\right)$ in phase space $H \times \Sigma$.

\section{The uniformly absorbing set}

Lemma 1 (Ref. 15) For every $\tau \in \mathbb{R}$, any nonnegative locally summable function $\phi$ on $R_{\tau}$ and for every $\beta>0$, we have

$$
\int_{\tau}^{t} \phi(s) \mathrm{e}^{-\beta(t-s)} \mathrm{d} s \leqslant \frac{1}{1-\mathrm{e}^{-\beta}} \sup _{\theta \geqslant \tau} \int_{\theta}^{\theta+1} \phi(s) \mathrm{d} s,
$$

for all $t \geqslant \tau$.

Lemma 2 Assume $f \in \Sigma \subset L_{b}^{2}(\mathbb{R} ; H), u_{\tau} \in H$. Then there exists a time

$$
T_{0}=\tau+\frac{1}{\lambda_{1} v_{0}} \ln \left(\frac{\left|u_{\tau}\right|^{2}}{\frac{4}{\lambda_{1} v_{0}}+\frac{4}{\lambda_{1}^{2} v_{0}^{2}}}\left\|f_{0}\right\|_{L_{b}^{2}(\mathbb{R} ; H)}^{2}\right)
$$


such that for any $t \geqslant T_{0}$, the process $U(t, \tau)$ possesses a bounded uniformly (w.r.t. $f \in \Sigma$ ) absorbing set $\mathfrak{B}$ in $H$, where $\mathfrak{B}=\left\{u \in H:|u| \leqslant C\left\|f_{0}\right\|_{L_{b}^{2}(R ; H)} \doteq R_{0}\right\}$ is a bounded set in $H$ which attracts all bounded subsets in $H$, where $R_{0}^{2}=\left(8 / \lambda_{1} v_{0}\right)\left(1+1 / \lambda_{1} v_{0}\right)\left\|f_{0}\right\|_{L_{b}^{2}(\mathbb{R} ; H)}^{2}$.

Proof: Taking the inner product of (1) with $u$, we derive the energy equation

$$
\frac{d}{\mathrm{~d} t}|u(t)|^{2}+2 v_{0}\|u(t)\|^{2}+2 v_{1}\|u(t)\|^{4}=2\langle f(t), u(t)\rangle,
$$

and thus

$$
\frac{d}{\mathrm{~d} t}|u(t)|^{2}+\lambda_{1} v_{0}|u(t)|^{2} \leqslant \frac{|f(t)|^{2}}{v_{0} \lambda_{1}} .
$$

From the Gronwall inequality, one can easily deduce

$$
\begin{aligned}
|u(t)|^{2} \leqslant\left|u_{\tau}\right|^{2} \mathrm{e}^{-\lambda_{1} v_{0}(t-\tau)} & \\
& +4 \mathrm{e}^{-\lambda_{1} v_{0} t} \int_{\tau}^{t} \frac{\mathrm{e}^{\lambda_{1} \nu_{0} \xi}}{v_{0} \lambda_{1}}|f(\xi)|^{2} \mathrm{~d} \xi .
\end{aligned}
$$

By Lemma 1, we derive

$$
\begin{aligned}
& |u(t)|^{2} \\
& \leqslant\left|u_{\tau}\right|^{2} \mathrm{e}^{-\lambda_{1} v_{0}(t-\tau)} \\
& +\frac{4}{\lambda_{1} v_{0}} \int_{\tau}^{t} \mathrm{e}^{\lambda_{1} v_{0}(\xi-t)}\|f(\xi)\|^{2} \mathrm{~d} \xi \\
& \leqslant\left|u_{\tau}\right|^{2} \mathrm{e}^{-\lambda_{1} v_{0}(t-\tau)}+\frac{4}{\lambda_{1} v_{0}}\left(\int_{t-1}^{t}|f(s)|^{2} \mathrm{~d} s\right. \\
& +\mathrm{e}^{-\lambda_{1} v_{0}} \int_{t-2}^{t-1}\|f(s)\|^{2} \mathrm{~d} s \\
& \left.+\mathrm{e}^{-2 \lambda_{1} v_{0}} \int_{t-3}^{t-2}|f(s)|^{2} \mathrm{~d} s+\cdots\right) \\
& \leqslant\left|u_{\tau}\right|^{2} \mathrm{e}^{-\lambda_{1} v_{0}(t-\tau)} \\
& +\frac{4}{\lambda_{1} v_{0}}\left(1+\mathrm{e}^{-\lambda_{1} v_{0}}+\mathrm{e}^{-2 \lambda_{1} v_{0}}+\cdots\right) \\
& \quad \times\|f(s)\|_{L_{b}^{2}(\mathbb{R} ; H)}^{2} \\
& \leqslant\left|u_{\tau}\right|^{2} \mathrm{e}^{-\lambda_{1} v_{0}(t-\tau)} \\
& +\frac{4}{\lambda_{1} v_{0}}\left(1+\frac{1}{\lambda_{1} v_{0}}\right)\|f(s)\|_{L_{b}^{2}(\mathbb{R} ; H)}^{2} \\
& \leqslant\left|u_{\tau}\right|^{2} \mathrm{e}^{-\lambda_{1} v_{0}(t-\tau)} \\
& +\frac{4}{\lambda_{1} v_{0}}\left(1+\frac{1}{\lambda_{1} v_{0}}\right)\left\|f_{0}\right\|_{L_{b}^{2}(\mathbb{R} ; H)}^{2}
\end{aligned}
$$

and choosing

$$
\left|u_{\tau}\right|^{2} \mathrm{e}^{-\lambda_{1} v_{0}(t-\tau)} \leqslant \frac{4}{\lambda_{1} v_{0}}\left(1+\frac{1}{\lambda_{1} v_{0}}\right)\left\|f_{0}\right\|_{L_{b}^{2}(\mathbb{R} ; H)}^{2},
$$

we deduce that there exists a time $T_{0}=\tau+\left(1 / \lambda_{1} v_{0}\right) \ln \left(\left(\left|u_{\tau}\right|^{2} /\left(\left(4 / \lambda_{1} v_{0}\right)+\right.\right.\right.$ $\left.\left.\left.4 / \lambda_{1}^{2} v_{0}^{2}\right)\right)\left\|f_{0}\right\|_{L_{b}^{2}(\mathbb{R} ; H)}^{2}\right)$, such that for $t \geqslant T_{0}$, the bounded set $\mathfrak{B}=\left\{u:|u|^{2} \leqslant R_{0}^{2}\right\}$ is a uniformly (w.r.t. $f \in \Sigma$ ) absorbing set for the process $\left\{U_{f}(t, \tau)\right\}$ in $H$, where

$$
R_{0}^{2}=\frac{8}{\lambda_{1} v_{0}}\left(1+\frac{1}{\lambda_{1} v_{0}}\right)\left\|f_{0}\right\|_{L_{b}^{2}(\mathbb{R} ; H)}^{2}, t \in \mathbb{R} .
$$

\section{The continuity of skew product flow}

From the Aubin-Lions compact argument and definition of symbol spaces, we can derive the continuity of process and skew product flow.

Lemma 3 For any $f \in \Sigma=\mathscr{H}\left(f_{0}\right)$, the family of processes $\left\{U_{f}(t, \tau)\right\}$ defined on $H$, corresponding to our problem is $(H \times \Sigma, H)$ continuous.

Proof: This is true by the property of a global solution which is continuous.

Lemma 4 Let $\left\{u_{\tau}^{n}\right\}$ be a sequence in $H$ converging to $u_{\tau} \in H$ in H-topology and $\left\{f^{n}\right\} \subset \Sigma$ be a sequence converging to $f \in \Sigma$. Then for any fixed $\tau \in \mathbb{R}$, it yields

(i) $U_{f^{n}}(\cdot, \tau) u_{\tau}^{n} \rightarrow U_{f}(\cdot, \tau) u_{\tau}$ weakly in $H$.

(ii) $U_{f^{n}}(t, \tau) u_{\tau}^{n} \rightarrow U_{f}(t, \tau) u_{\tau}$ weakly in $L^{2}(\tau, T ; V)$.

(iii) $U_{f^{n}}(t, \tau) u_{\tau}^{n} \rightarrow U_{f}(t, \tau) u_{\tau}$ weakly in $L^{4}(\tau, T ; V)$.

Proof: Let $u_{n}(t)=U_{f^{n}}(t, \tau) u_{\tau}^{n}$ and $u(t)=U_{f}(t, \tau) u_{\tau}$ for $t \geqslant \tau$. From the existence of a global weak solution, we deduce that

$\left\{u_{n}\right\}_{n}$ is bounded in $L^{\infty}([\tau, \infty) ; H) \cap L^{2}(\tau, T ; V)$, $\forall T>\tau$. (11)

Hence since $u_{n}^{\prime}=f(t)-v_{0} A u_{n}-\mathbb{A} u_{n}+B\left(u_{n}\right)$, it follows that $\left\{u_{n}^{\prime}\right\}_{n}$ is bounded in $L^{2}\left(\tau, T ; V^{\prime}\right)$ for all $T>0$. Then for all $v \in V$ and $\tau \leqslant t \leqslant t+a \leqslant T$, by the Cauchy-Schwarz inequality, we obtain

$$
\begin{aligned}
\left(u_{n}(t+a)-u_{n}(t), v\right) & =\int_{t}^{t+a}\left\langle u_{n}^{\prime}(s), v\right\rangle \mathrm{d} s \\
& \leqslant c_{T}\|v\| a^{1 / 2},
\end{aligned}
$$

where $c_{T}>0$ is independent of $n$. Choosing $v=$ $u_{n}(t+a)-u_{n}(t) \in V$ for almost every $t$, we have

$$
\left|u_{n}(t+a)-u_{n}(t)\right|^{2} \leqslant c_{T} a^{1 / 2}\left\|u_{n}(t+a)-u_{n}(t)\right\|,
$$


and hence

$$
\begin{aligned}
& \int_{t}^{t+a}\left|u_{n}(t+a)-u_{n}(t)\right|^{2} \mathrm{~d} t \\
& \quad \leqslant c_{T} a^{1 / 2} \int_{t}^{t+a}\left\|u_{n}(t+a)-u_{n}(t)\right\| \mathrm{d} t .
\end{aligned}
$$

Using (11) and (13) we obtain

$$
\int_{t}^{t+a}\left|u_{n}(t+a)-u_{n}(t)\right|^{2} \mathrm{~d} t \leqslant \bar{c}_{T} a^{1 / 2}
$$

for another positive constant $\bar{c}_{T}$ independent of $n$. Hence

$$
\limsup _{a \rightarrow 0} \int_{t}^{t+a}\left|u_{n}(t+a)-u_{n}(t)\right|^{2} \mathrm{~d} t=0, \quad \forall r>0 .
$$

Then from (11) and by a diagonal process, we can extract a subsequence $\left\{u_{n^{\prime}}\right\}_{n^{\prime}}$ such that

$$
\begin{aligned}
& u_{n^{\prime}} \rightarrow \tilde{u} \text { weakly in } L^{\infty}([\tau, \infty) ; H), \text { as } n^{\prime} \rightarrow \infty, \\
& u_{n^{\prime}} \rightarrow \tilde{u} \text { weakly in } L_{\text {loc }}^{2}([\tau, \infty) ; V) \text {, as } n^{\prime} \rightarrow \infty,
\end{aligned}
$$

and $u_{n^{\prime}} \rightarrow \tilde{u}$ strongly in $L_{\text {loc }}^{2}([\tau, \infty) ; H)$ as $n^{\prime} \rightarrow$ $\infty$ for all $r>0$, for some $\tilde{u} \in L^{\infty}([\tau, \infty) ; H) \cap$ $L_{\text {loc }}^{2}([\tau, \infty) ; V)$. By the uniqueness of solutions, it yields $\tilde{u}=u$, i.e., $\left\{u_{n}\right\}_{n}$ converges to $u$ weakly in $L_{\text {loc }}^{2}([\tau, \infty) ; V)$. Hence for all $v \in E$, it follows that

$$
\left(u_{n}(t), v\right) \rightarrow(u(t), v) \text {, for a.e. } t \geqslant \tau .
$$

Furthermore, from (11) and (12), we see that $\left\{\left(u_{n}(t), v\right)\right\}_{n}$ is equibounded and equicontinuous on $[\tau, T]$ for all $T>\tau$. Hence

$$
\left(u_{n}(t), v\right) \rightarrow(u(t), v) \text {, for all } t \geqslant \tau .
$$

Since $E$ is dense in $H$, the lemma holds. By the energy inequality, we can prove (iii) by a similar technique.

\section{The uniformly asymptotic compactness of a process}

Lemma 5 For any $f \in \Sigma$, the family of processes $\left\{U_{f}(t, \tau)\right\}, f \in \Sigma$ defined on $H$ to problem (1) is asymptotically compact in $H$.

Proof: Let $\left\{u_{\tau}^{n}\right\}$ be a bounded sequence in $H$ and $\left\{f^{n}\right\}$ in $\Sigma$ converge to $u_{\tau}$ and $f$, respectively, as $n \rightarrow$ $\infty$.

From Lemma 2, we see that for any fixed $u_{\tau} \in H$ and $\tau \in \mathbb{R}$, there exists a time $T_{0}=T_{0}(\tau)$ such that for all $t_{n} \geqslant T_{0},\left\{U_{f^{n}}\left(t_{n}, \tau\right) u_{\tau}^{n}\right\} \subseteq \mathfrak{B},\left\{U_{f^{n}}\left(t_{n}, \tau\right) u_{\tau}^{n}\right\}$ is weakly relatively compact in $H$ and

$$
U_{f^{n}}\left(t_{n}, \tau\right) u_{\tau}^{n} \rightarrow u \text { weakly in } H \text { as } n \rightarrow \infty
$$

for some $u \in H$ and convergent subsequence. Similarly, for each $T>0$ and $t_{n} \geqslant T_{0}+T$, we also have $u_{T}^{n} \doteq U_{f^{n}}\left(t_{n}-T, \tau\right) u_{\tau}^{n} \rightarrow u_{T}$ weakly in $H$ as $n \rightarrow \infty$

for some $u_{T} \in H$.

Noting the property of translation semigroup $\{T(t): t \geqslant \tau\}$ which satisfies

$$
U_{T(h) f}(t, \tau)=U_{f}(t+h, \tau+h), \quad \forall h \geqslant 0, f \in \Sigma,
$$

then for any $t_{n}-T \geqslant \tau$, we derive that

$$
\begin{aligned}
U_{f^{n}}\left(t_{n}, \tau\right) & =U_{f^{n}}\left(t_{n}, t_{n}-T\right) U_{f^{n}}\left(t_{n}-T, \tau\right) \\
& =U_{T\left(t_{n}-T\right) f^{n}}(T, 0) U_{f^{n}}\left(t_{n}-T, \tau\right) .
\end{aligned}
$$

Denoting $f_{T}^{n}=T\left(t_{n}-T\right) f^{n}$, by (18) and (19),

$$
\begin{aligned}
U_{f^{n}}\left(t_{n}, \tau\right) u_{\tau}^{n} & =U_{f_{T}^{n}}(T, 0) U_{f^{n}}\left(t_{n}-T, \tau\right) u_{\tau}^{n} \\
& =U_{f_{T}^{n}}(T, 0) u_{T}^{n}, \quad t_{n}-T \geqslant \tau .
\end{aligned}
$$

Since $\left\{f_{T}^{n}\right\} \subset \Sigma$ and $\Sigma$ is compact in $L_{\text {loc }}^{2}(\mathbb{R} ; H)$, there exists a subsequence of $\left\{f_{T}^{n}\right\}$ (also denoted by $\left\{f_{T}^{n}\right\}$ ) and some $f_{T} \in \Sigma$ such that

$f_{T}^{n} \rightarrow f_{T}$ strongly in $L_{\text {loc }}^{2}(\mathbb{R} ; H)$ as $n \rightarrow \infty, \forall T>0$.

By Lemma 4, (16), (17), (20), and (21) we obtain $u=U_{f_{T}}(T, 0) u_{T}$ for all $T>0$.

In the following, we shall prove the asymptotic compactness in $H$ via the energy equation method, i.e., $\left|U_{f_{T}^{n}}\left(t_{n}, \tau\right) u_{\tau}^{n}-u\right| \rightarrow 0$ as $n \rightarrow \infty$. To achieve this goal, we need to prove the upper and lower continuity of the processes

$$
\liminf _{n}\left|U_{f^{n}}\left(t_{n}, \tau\right) u_{\tau}^{n}\right|=\liminf _{n}\left|U_{f_{T}^{n}}(T, 0) u_{T}^{n}\right| \geqslant|u|,
$$

$\underset{n}{\limsup }\left|U_{f^{n}}\left(t_{n}, \tau\right) u_{\tau}^{n}\right|=\underset{n}{\limsup }\left|U_{f_{T}^{n}}(T, 0) u_{T}^{n}\right| \leqslant|u|$.

The weak convergence for the process ensures that (22) is true. What we need next is to prove (23) from the weak and norm convergence of the corresponding sequences.

Taking the inner product of (5) with $\mathrm{e}^{2 \lambda_{1} v_{0} t} u(t)$ in $H$, we derive that

$$
\begin{aligned}
& \frac{1}{2} \frac{\mathrm{d}}{\mathrm{d} t} \mathrm{e}^{2 \lambda_{1} v_{0} t}(u, u)+v_{0} \mathrm{e}^{2 \lambda_{1} v_{0} t}(A u(t), u(t)) \\
&+v_{1} \mathrm{e}^{2 \lambda_{1} v_{0} t}(\mathbb{A} u, u) \\
&=\lambda_{1} v_{0} \mathrm{e}^{2 \lambda_{1} v_{0} t}(u, u)+\mathrm{e}^{2 \lambda_{1} v_{0} t}(f, u(t)),
\end{aligned}
$$


integrating (24) over $[\tau, t]$ yields

$$
\begin{aligned}
& (u(t), u(t))=(u(\tau), u(\tau)) \mathrm{e}^{-2 \lambda_{1} v_{0} t} \\
& +2 \int_{\tau}^{t} \mathrm{e}^{-2 \lambda_{1} v_{0}(t-s)}(f(s), u(s)) \mathrm{d} s \\
& \quad-2 \int_{\tau}^{t} v_{0} \mathrm{e}^{-2 \lambda_{1} v_{0}(t-s)}(A u(s), u(s)) \mathrm{d} s \\
& -2 \int_{\tau}^{t} v_{1} \mathrm{e}^{-2 \lambda_{1} v_{0}(t-s)}(\mathbb{A}(s), u(s)) \mathrm{d} s \\
& +2 \lambda_{1} v_{0} \int_{\tau}^{t} \mathrm{e}^{-2 \lambda_{1} v_{0}(t-s)}(u(s), u(s)) \mathrm{d} s
\end{aligned}
$$

for all $t \geqslant \tau, \tau \in \mathbb{R}$.

Applying (25) to $U_{f_{T}^{n}}(T, 0) u_{T}^{n}$, we derive that

$$
\begin{aligned}
\left(U_{f_{T}^{n}}(T, 0)\right. & \left.u_{T}^{n}, U_{f_{T}^{n}}(T, 0) u_{T}^{n}\right)=\left(u_{T}^{n}, u_{T}^{n}\right) \mathrm{e}^{-2 \lambda_{1} v_{0} T} \\
+ & 2 \int_{\tau}^{T} \mathrm{e}^{-2 \lambda_{1} v_{0}(T-s)} \\
& \times\left(f_{s}^{n}(s), U_{f_{s}^{n}}(s, 0) u_{s}^{n}\right) \mathrm{d} s \\
- & \left(2 \int_{\tau}^{T} v_{0} \mathrm{e}^{-2 \lambda_{1} v_{0}(T-s)}\right. \\
& \times\left(A U_{f_{s}^{n}}(s, 0) u_{s}^{n}, U_{f_{s}^{n}}(s, 0) u_{s}^{n}\right) \mathrm{d} s \\
& +2 \int_{\tau}^{T} v_{1} \mathrm{e}^{-2 \lambda_{1} v_{0}(T-s)} \\
& \left.\times\left(\mathbb{A} U_{f_{s}^{n}}(s, 0) u_{s}^{n}, U_{f_{s}^{n}}(s, 0) u_{s}^{n}\right) \mathrm{d} s\right) \\
+ & 2 \lambda_{1} v_{0} \int_{\tau}^{T} \mathrm{e}^{-2 \lambda_{1} v_{0}(T-s)} \\
& \times\left(U_{f_{s}^{n}}(s, 0) u_{s}^{n}, U_{f_{s}^{n}}(s, 0) u_{s}^{n}\right) \mathrm{d} s \\
= & I_{2}+I_{3}+I_{4} .
\end{aligned}
$$

From Lemma 2, we deduce that

$$
\limsup _{n} I_{1}=\limsup _{n}\left(u_{T}^{n}, u_{T}^{n}\right) \mathrm{e}^{-2 \lambda_{1} v_{0} T} \leqslant R_{0}^{2} \mathrm{e}^{-2 \lambda_{1} v_{0} T} \text {. }
$$

By Lemma 4,

$U_{f_{s}^{n}}(s, 0) u_{s}^{n} \rightarrow U_{f_{s}}(s, 0) u_{s}$ weakly in $H, L^{2}(\tau, T ; V)$,

$$
\forall T>\tau \text {. }
$$

Hence we deduce from (26) and (28) that

$$
\begin{aligned}
\lim _{n \rightarrow \infty} I_{2} & =\lim _{n \rightarrow \infty} 2 \int_{\tau}^{T} \mathrm{e}^{-2 \lambda_{1} v_{0}(T-s)}\left(f_{s}^{n}(s), U_{f_{s}^{n}}(s, 0) u_{s}^{n}\right) \mathrm{d} s \\
& =2 \int_{\tau}^{T} \mathrm{e}^{-2 \lambda_{1} v_{0}(T-s)}\left(f_{s}(s), U_{f_{s}}(s, 0) u_{s}\right) \mathrm{d} s
\end{aligned}
$$

and

$$
\begin{aligned}
\lim _{n \rightarrow \infty} I_{4}= & \lim _{n \rightarrow \infty} 2 \lambda_{1} v_{0} \int_{\tau}^{T} \mathrm{e}^{-2 \lambda_{1} v_{0}(T-s)} \\
& \times\left(U_{f_{s}^{n}}(s, 0) u_{s}^{n}, U_{f_{s}^{n}}(s, 0) u_{s}^{n}\right) \mathrm{d} s \\
= & 2 \lambda_{1} v_{0} \int_{\tau}^{T} \mathrm{e}^{-2 \lambda_{1} v_{0}(T-s)} \\
& \times\left(U_{f_{s}}(s, 0) u_{s}, U_{f_{s}}(s, 0) u_{s}\right) \mathrm{d} s .
\end{aligned}
$$

Next, we want to deal with the third term on the right-hand side of (26). In fact, since $A$ is a bounded linear operator from $V$ into $V^{\prime}, \mathbb{A}: V \rightarrow V^{\prime}$ is bounded. From Lemma 4 we obtain

$$
\begin{aligned}
\limsup _{n} & \left(-2 \int_{\tau}^{T} v_{0} \mathrm{e}^{-2 \lambda_{1} v_{0}(T-s)}\right. \\
& \left.\times\left(A U_{f_{s}^{n}}(s, 0) u_{s}^{n}, U_{f_{s}^{n}}(s, 0) u_{s}^{n}\right) \mathrm{d} s\right) \\
=- & \liminf _{n}\left(2 \int_{\tau}^{T} v_{0} \mathrm{e}^{-2 \lambda_{1} v_{0}(T-s)}\right. \\
& \left.\times\left\|U_{f_{s}^{n}}(s, 0) u_{s}^{n}\right\|^{2} \mathrm{~d} s\right) \\
\leqslant- & \left(2 \int_{\tau}^{T} v_{0} \mathrm{e}^{-2 \lambda_{1} v_{0}(T-s)}\left\|U_{f_{s}}(s, 0) u_{s}\right\|^{2} \mathrm{~d} s\right) \\
=- & 2 \int_{\tau}^{T} v_{0} \mathrm{e}^{-2 \lambda_{1} v_{0}(T-s)} \\
& \times\left(A U_{f_{s}}(s, 0) u_{s}, U_{f_{s}}(s, 0) u_{s}\right) \mathrm{d} s
\end{aligned}
$$

and

$$
\begin{aligned}
\underset{n}{\limsup }\left(-2 \int_{\tau}^{T} v_{1} \mathrm{e}^{-2 \lambda_{1} v_{0}(T-s)}\right. & \left.\times\left(\mathbb{A} U_{f_{s}^{n}}(s, 0) u_{s}^{n}, U_{f_{s}^{n}}(s, 0) u_{s}^{n}\right) \mathrm{d} s\right) \\
=- & \liminf _{n}\left(2 \int_{\tau}^{T} v_{1} \mathrm{e}^{-2 \lambda_{1} v_{0}(T-s)}\right. \\
& \left.\times\left\|U_{f_{s}^{n}}(s, 0) u_{s}^{n}\right\|^{4} \mathrm{~d} s\right) \\
\leqslant- & 2 \int_{\tau}^{T} v_{1} \mathrm{e}^{-2 \lambda_{1} v_{0}(T-s)}\left\|U_{f_{s}}(s, 0) u_{s}\right\|^{4} \mathrm{~d} s \\
=-2 & \int_{\tau}^{T} v_{1} \mathrm{e}^{-2 \lambda_{1} v_{0}(T-s)} \\
& \times\left(\mathbb{A} U_{f_{s}}(s, 0) u_{s}, U_{f_{s}}(s, 0) u_{s}\right) \mathrm{d} s .
\end{aligned}
$$

Hence

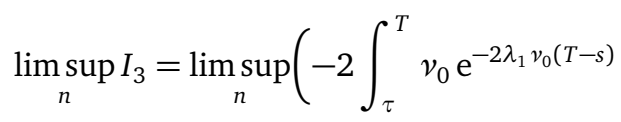




$$
\begin{aligned}
\times & \left(A U_{f_{s}^{n}}(s, 0) u_{s}^{n}, U_{f_{s}^{n}}(s, 0) u_{s}^{n}\right) \mathrm{d} s \\
- & 2 \int_{\tau}^{T} v_{1} \mathrm{e}^{-2 \lambda_{1} v_{0}(T-s)} \\
& \left.\times\left(\mathbb{A} U_{f_{s}^{n}}(s, 0) u_{s}^{n}, U_{f_{s}^{n}}(s, 0) u_{s}^{n}\right) \mathrm{d} s\right) \\
=- & \liminf _{n}\left(2 \int_{\tau}^{T} v_{0} \mathrm{e}^{-2 \lambda_{1} v_{0}(T-s)}\right. \\
& \times\left(A U_{f_{s}^{n}}(s, 0) u_{s}^{n}, U_{f_{s}^{n}}(s, 0) u_{s}^{n}\right) \mathrm{d} s \\
+ & 2 \int_{\tau}^{T} v_{1} \mathrm{e}^{-2 \lambda_{1} v_{0}(T-s)} \\
& \left.\times\left(\mathbb{A} U_{f_{s}^{n}}(s, 0) u_{s}^{n}, U_{f_{s}^{n}}(s, 0) u_{s}^{n}\right) \mathrm{d} s\right) \\
\leqslant-\left(2 \int_{\tau}^{T} v_{0} \mathrm{e}^{-2 \lambda_{1} v_{0}(T-s)}\right. & \times\left(A U_{f_{s}}(s, 0) u_{s}, U_{f_{s}}(s, 0) u_{s}\right) \mathrm{d} s \\
+ & 2 \int_{\tau}^{T} v_{1} \mathrm{e}^{-2 \lambda_{1} v_{0}(T-s)} \\
& \left.\times\left(\mathbb{A} U_{f_{s}}(s, 0) u_{s}, U_{f_{s}}(s, 0) u_{s}\right) \mathrm{d} s\right) .
\end{aligned}
$$

Combining (26) and (27)-(30), we conclude that

$$
\begin{aligned}
& \underset{n}{\limsup }\left(U_{f_{T}^{n}}(T, 0) u_{T}^{n}, U_{f_{T}^{n}}(T, 0) u_{T}^{n}\right) \leqslant R_{0}^{2} \mathrm{e}^{-2 \lambda_{1} v_{0} T} \\
& \quad+2 \int_{\tau}^{T} \mathrm{e}^{-2 \lambda_{1} v_{0}(T-s)}\left(f_{s}(s), U_{f_{s}}(s, 0) u_{s}\right) \mathrm{d} s \\
& -\left(2 \int_{\tau}^{T} v_{0} \mathrm{e}^{-2 \lambda_{1} v_{0}(T-s)}\left(A U_{f_{s}}(s, 0) u_{s}, U_{f_{s}}(s, 0) u_{s}\right) \mathrm{d} s\right. \\
& \left.+2 \int_{\tau}^{T} v_{1} \mathrm{e}^{-2 \lambda_{1} v_{0}(T-s)}\left(\mathbb{A} U_{f_{s}}(s, 0) u_{s}, U_{f_{s}}(s, 0) u_{s}\right) \mathrm{d} s\right) \\
& +2 \lambda_{1} v_{0} \int_{\tau}^{T} \mathrm{e}^{-2 \lambda_{1} v_{0}(T-s)}\left(U_{f_{s}}(s, 0) u_{s}, U_{f_{s}}(s, 0) u_{s}\right) \mathrm{d} s .
\end{aligned}
$$

By the same technique as above, applying (25) to $u=U_{f_{T}}(T, 0) u_{T}$ yields

$$
\begin{aligned}
& \left(U_{f_{T}}(T, 0) u_{T}, U_{f_{T}}(T, 0) u_{T}\right)=\left(u_{T}, u_{T}\right) \mathrm{e}^{-2 \lambda_{1} v_{0} T} \\
& \quad+2 \int_{\tau}^{T} \mathrm{e}^{-2 \lambda_{1} v_{0}(T-s)}\left(f_{s}(s), U_{f_{s}}(s, 0) u_{s}\right) \mathrm{d} s \\
& -\left(2 \int_{\tau}^{T} v_{0} \mathrm{e}^{-2 \lambda_{1} v_{0}(T-s)}\left(A U_{f_{s}}(s, 0) u_{s}, U_{f_{s}}(s, 0) u_{s}\right) \mathrm{d} s\right. \\
& \left.+2 \int_{\tau}^{T} v_{1} \mathrm{e}^{-2 \lambda_{1} v_{0}(T-s)}\left(\mathbb{A} U_{f_{s}}(s, 0) u_{s}, U_{f_{s}}(s, 0) u_{s}\right) \mathrm{d} s\right)
\end{aligned}
$$

$$
+2 \lambda_{1} v_{0} \int_{\tau}^{T} \mathrm{e}^{-2 \lambda_{1} v_{0}(T-s)}\left(U_{f_{s}}(T, 0) u_{s}, U_{f_{s}}(s, 0) u_{s}\right) \mathrm{d} s .
$$

It follows from (32), (33), and (17) that for any $T>$ $\tau$,

$$
\begin{aligned}
& \underset{n}{\limsup }\left(U_{f_{T}^{n}}(T, 0) u_{T}^{n}, U_{f_{T}^{n}}(T, 0) u_{T}^{n}\right) \\
& \quad \leqslant\left(u_{T}, u_{T}\right)+R_{0}^{2} \mathrm{e}^{-2 \lambda_{1} v_{0} T}-\left(u_{T}^{n}, u_{T}^{n}\right) \mathrm{e}^{-2 \lambda_{1} v_{0} T} \\
& \quad \leqslant\left(U_{f_{T}}(T, 0) u_{T}, U_{f_{T}}(T, 0) u_{T}\right)+R_{0}^{2} \mathrm{e}^{-2 \lambda_{1} v_{0} T} .
\end{aligned}
$$

Hence letting $T$ tend to $\infty$, we derive that

$$
\begin{aligned}
& \lim _{n \rightarrow \infty}\left|U_{f^{n}}\left(t_{n}, \tau\right) u_{\tau}^{n}\right|^{2} \\
& \quad=\limsup _{n, T \rightarrow \infty}\left|U_{f_{T}^{n}}(T, 0) u_{T}^{n}\right|^{2} \\
& \quad=\limsup _{n, T \rightarrow \infty}\left(U_{f_{T}^{n}}(T, 0) u_{T}^{n}, U_{f_{T}^{n}}(T, 0) u_{T}^{n}\right) \\
& \quad \leqslant \lim _{T \rightarrow \infty}\left(U_{f_{T}}(T, 0) u_{T}, U_{f_{T}}(T, 0) u_{T}\right)=|u|^{2} .
\end{aligned}
$$

From (22),

$$
\limsup _{n, T \rightarrow \infty}\left|U_{f_{T}^{n}}(T, 0) u_{T}^{n}\right|^{2}=\lim _{n \rightarrow \infty}\left|U_{f^{n}}\left(t_{n}, \tau\right) u_{\tau}^{n}\right|^{2}=|u|^{2} .
$$

Combining the norm convergence (34) and weak convergence (16) we conclude that

$$
\lim _{n \rightarrow \infty}\left|U_{f_{T}^{n}}\left(t_{n}, \tau\right) u_{\tau}^{n}-u\right|^{2}=0,
$$

which implies the lemma is true.

Lemma 6 The family of processes $\left\{U_{f}(t, \tau)\right\}, f \in \Sigma$ defined on $H$ for problem (1) is uniformly (w.r.t. $f \in$ $\Sigma)$ asymptotically compact in $H$-topology.

Proof: From the theory in Ref. 15, to achieve the uniformly asymptotic compactness of a process, what we need is to verify that $\left\{U_{f}(t, \tau)\right\}$ possesses a compact uniformly (w.r.t. $f \in \Sigma$ ) attracting set in $H$.

For all uniformly bounded absorbing sets $\mathfrak{B}$ in $H$, we can construct the $\omega$-limit set as

$$
\omega_{\tau, \Sigma}(\mathfrak{B}) \doteq \bigcap_{t \geqslant \tau} \overline{\bigcup_{f \in \Sigma} \bigcup_{s \geqslant t} U_{f}(s, \tau) \mathfrak{B}},
$$

where $\overline{(\cdot)}$ denotes the closure topology in $H$. From the definition of $\omega$-limit set, we see that

$$
\begin{gathered}
u \in \omega_{\tau, \Sigma}(\mathfrak{B}) \Leftrightarrow \exists \text { sequences }\left\{u^{n}\right\} \subset \mathfrak{B}, \\
\left\{f^{n}\right\} \subset \Sigma, \quad\left\{t_{n}\right\} \subset[\tau, \infty) \text {, such that } \\
\lim _{n \rightarrow \infty} t_{n}=\infty, U_{f^{n}}\left(t_{n}, \tau\right) u^{n} \rightarrow u \\
\text { weakly in } H \text { as } n \rightarrow \infty .
\end{gathered}
$$


The existence of uniformly an absorbing set implies $\omega_{\tau, \Sigma}(\mathfrak{B})$ is independent of $\tau$. We claim that $\omega_{\tau, \Sigma}(\mathfrak{B})$ is a compact uniformly (w.r.t. $f \in \Sigma$ ) attracting set for the process $\left\{U_{f}(t, \tau)\right\}$ in $H$. Combining the uniformly (w.r.t. $f \in \Sigma$ ) absorbing property (Lemma 2), the asymptotic compactness property of the process $\left\{U_{f}(t, \tau)\right\}, f \in \Sigma$ in $H$ (Lemma 5), we conclude that $\omega_{\tau, \Sigma}(\mathfrak{B})$ is a nonempty compact set in $H$.

For any bounded subset $B \in H$, we obtain the following property from Lemma 2 :

$$
\lim _{t \rightarrow \infty} \sup _{f \in \Sigma} \operatorname{dist}_{H}\left(U_{f}(t, \tau) B, \omega_{\tau, \Sigma}(\mathfrak{B})\right)=0,
$$

which also means that

$$
\omega_{\tau, \Sigma}(B) \subseteq \omega_{\tau, \Sigma}(\mathfrak{B}) .
$$

Hence (35) and (36) imply the uniformly (w.r.t. $f \in \Sigma$ ) attracting property of $\omega_{\tau, \Sigma}(\mathfrak{B})$ in $H$, and the uniformly asymptotic compactness of the process holds.

\section{The global and uniform attractors}

From Lemmas 2-6, we summarize the properties of a process as follows: (i) $\left\{U_{f}(t, \tau)\right\}$ is continuous in $H$; (ii) the process possesses a uniformly absorbing set in $H$; (iii) the process is uniformly asymptotically compact in $H$ with respect to $f \in \Sigma$. Combining the proposition of translation semigroup and symbol spaces, we can show that the skew product flow is continuous and attracting which satisfies the compact property, so we have the existence of global and uniform attractors for the skew product flow and process, respectively, as in the following.

Theorem 4 Let $f \in \Sigma$ be the symbols, $u_{0} \in H$. Then the global weak solution for problem (1) generates a family of processes $\left\{U_{f}(t, \tau)\right\}(f \in \Sigma)$ and skew product flow $S(t)=\left(U_{f}(t, \tau), T(t)\right) \in H \times \Sigma$. If the symbol spaces are chosen as translation compact, bounded or normal class functional spaces, the skew product flow possesses a compact global attractor $\mathscr{A}$ in $H \times \Sigma$ :

$$
\mathscr{A}=\bigcup_{\sigma \in \Sigma} \mathscr{K}_{\sigma}(\tau) \times\{\sigma\},
$$

where $\mathscr{K}_{\sigma}(\tau)$ denotes all bounded complete trajectories under the process and translation semigroup. Furthermore, let $\Pi_{1}$ and $\Pi_{2}$ be two projections from $H \times \Sigma$ to $H$ and $\Sigma$, respectively. Then

$$
\Pi_{1} \mathscr{A}=\mathscr{A}_{\Sigma}=\bigcup_{\sigma \in \Sigma} \mathscr{K}_{\sigma}(\tau)
$$

is the uniform attractor of process in $H$, and $\Pi_{1} \mathscr{A}=$ $\mathfrak{U}=\Sigma$ is the global attractor of the translation semigroup (also as shift operator) $T(\cdot)$.

Proof: Based on the continuity of a process (Lemma 3) and the shift operator, the existence of a uniformly absorbing set, and the uniform asymptotic compactness of a process, by the theory in Ref. 15, we can prove our result.

\section{Further research}

For this Ladyzhenskaya model, as an approximation to the 3D classical incompressible Navier-Stokes equations, which has some similar properties to the non-Newtonian fluid model, some further interesting problems can also be considered. (a) If the domain is unbounded, does the uniform attractor exist? (b) For the 2D Navier-Stokes equation, the weak solution can be regularized to a strong solution with a periodic boundary, and hence a weak attractor has more regularity, but what about this Ladyzhenskaya model? (c) The continuity of the attractor as $v_{1} \rightarrow 0$ may occur, but in which topology?

Acknowledgements: This work was in part supported by the Mainstay Fund from Henan Normal University. Xinguang Yang was also partially supported by NSFC (No.11726626) and the Key Project of Science and Technology of Henan Province (No. 182102410069). Yongqiang Chen was partly supported by the NSFC (11671122), the Young Teacher Research Fund of Henan Normal University (qd16152), the Project of Science and Technology in Henan Province (172102210342), and the Key Project of Education Department in Henan Province (17A120003).

\section{REFERENCES}

1. Ladyzhenskaya OA (1968) The Mathematical Theory of Viscous Incompressible Flow, 2nd edn, Gordon and Breach, New York.

2. Leray J (1933) Etude de diverses equations integrales nonlineaires et de quelques problèmes que pose l'hydrodynamique. J Math Pure Appl 12, 1-82.

3. Leray J (1934) Essai sur les mouvements plans d'un liquide visqueux que limitent des parois. J Math Pure Appl 13, 331-418.

4. Leray J (1934) Essai sur le mouvement d'un liquide visqueux emplissant l'espace. Acta Math 63, 193-248.

5. Hopf E (1951) Über die Anfangswertaufgable für die hydrodynamischen Grundgleichungen. Math Nachr 4, 213-31.

6. Temam R (1979) Navier-Stokes Equations, Theory and Numerical Analysis, North-Holland. 
7. Foias C, Manley O, Rosa R, Temam R (2001) NavierStokes Equations and Turbulence, Cambridge Univ Press.

8. Robinson JC (2013) Attractors and finitedimensional behaviour in the 2D Navier-Stokes equations. ISRN Math Anal 2013, 291823.

9. Lions JL, Prodi G (1959) Un théorème d'existence et unicité dans les equations de Navier-Stokes en dimension 3. Compte Rendus Acad Sci Paris 248, 319-21.

10. Lions JL (1969) Quelques Méthodes de Résolution Des Problémes Aux Limites Non Linéaires, Dunod, Paris.

11. Araújo GM, Milla Miranda M, Medeiros LA (2007) On the Navier-Stokes equations with variable viscosity in a noncylindrical domain. Appl Anal 86, 287-313.

12. Bezerra FDM, da Silva SH, Yang X (2018) Pullback attractor for the 3D Navier-Stokes flow with variable viscosity on some unbounded domains. Submitted.

13. Yang X, Feng B, Wang S, Ma TF (2017) Pullback dynamics of a 3D Navier-Stokes equation with nonlinear viscosity. Preprint.

14. Temam R (1997) Infinite Dimensional Dynamical Systems in Mechanics and Physics, 2nd edn, Springer, Berlin.

15. Chepyzhov VV, Vishik MI (2001) Attractors for Equations of Mathematical Physics, American Mathematical Society.

16. Lu S, Wu H, Zhong C (2005) Attractors for nonautonomous $2 D$ Navier-Stokes equations with normal external forces. Discrete Cont Dyn Syst 13, 701-19. 